\title{
Rethinking Fashion Therapy: Theoretical and Practical Foundations for Value Creations in Clothing and Textiles Discipline
}

\author{
Hyungjin Son ${ }^{1)}$, Sunwoo Kim ${ }^{1)}$, Yuri Lee ${ }^{1)}$, Jihyun Lee ${ }^{2)}$, Saeeun Lee ${ }^{1)}$ \\ ${ }^{1)}$ Seoul National University/ Research Institute of Human Ecology at SNU, South Korea \\ 2) Sejong University, South Korea
}

Keywords: fashion therapy, academic protocol, value creation

\section{Introduction}

Using and shopping for fashion products are expected to have therapeutic effects although many studies dealing with body image, physical appearance management, media effect, and irrational consumption in clothing and textiles (hereafter CT) tend to underscore the negative side of fashion-consciousness rather than the positive side. As a type of psychotherapy, fashion therapy (hereafter FT) improves mental health to enhance self-concepts through grooming behaviors in all parts of the human body including physical appearance management behaviors and fashion product consumption (Horn \& Gurel, 1981; Thompson, 1962). Until now, similar disciplines, such as psychology, women's studies, and art therapy studies, have provided academic and empirical grounds for FT studies.

Based on the literature reviews, this study suggests academic reasons why CT should prosper FT studies. In addition, we offer academic protocols for FT studies to systematize the research scopes and methodologies. Last, this study proposes how CT could create various values through FT studies in the academic, economic, and social perspectives.

\section{Theoretical and Practical Foundations of Fashion Therapy Research}

Previous studies in CT have revealed the negative issues related to fashion such as negative body image, distorted ideal body, negative media effects, and irrational consumption like impulsive or excessive buying (Han et al., 1991; Johnson et al., 2014; Kim \& Lennon, 2007). However, current psychological/behavioral studies in CT have discovered the positive side of fashion phenomena that could be applied for psychotherapeutic purposes (Rudd \& Carter, 2006; Wood-Barcalow et al.,2010). This new stream could facilitate positive public perception and value creations of CT. In effect, relevant academic disciplines of CT, such as psychology, women's studies, and art therapy, proposed theoretical backgrounds and empirical evidences of FT and intensified academia-field link for practical value creations (Castillo-Pérez et al., 2010; Graham \& Kligman, 1984; Steiner-Adair et al., 2002). With these reasons, CT should explore FT studies to take academic, economic, and social values.

\section{Academic Protocols of Fashion Therapy Research}

When conducting FT studies in CT, academic protocols should be developed considering the following points. First, FT studies should encompass a wide range of research scopes; whereas traditional fashion therapy studies have been confined to limited research areas such as beauty product consumption, and retail effects (Kang \& Johnson, 2011; Yoon \& Lee, 2012). Indeed, there are various physical appearance management behaviors that have positive effects through the clothes, skin care, makeup, weight control, and tattoos. Additionally, in the fashion product consumption process, FT studies should embrace more segmentized steps like information search, exploration, buying, and evaluation as research topics. Second, CT should expand research objects toward general public to improve academic/empirical values since the public could experience fashion on a daily basis; whereas, FT research has focused on mentally or physically disordered groups suffering from eating disorders, depression, or cancer (Dubler \& Gurel, 1984; Graham \& Kligman, 1984; Steiner-Adair et al., 2002; Yu \& Son, 2010). Third, since fashion behaviors influence human being's attitudes, behaviors, and 
emotions, future studies should apply diverse variables to demonstrate FT effects. Preceding studies have usually adopted psychological variables such as self-efficacy, self-esteem, and body image (Graham \& Kligman, 1984; Lee \& Kim, 2010), which could not thoroughly illustrate FT's therapeutic effects. Fourth, FT studies should expand research methods including quantitative/qualitative, and newly rising methods; whereas qualitative method has been mainly applied for medical cases in other disciplines (Graham \& Kligman, 1984; Paulson, 2008). CT, firmly establishing academic structures in psychosocial and cultural aspects, could execute quantitative method to verify hypotheses regarding concrete academic backgrounds. Furthermore, newly rising methods like neuro-imaging, psychophysiology, and eye-tracking methods could illustrate subjects' reaction to therapy or could complement existing methods. Last, we need to develop practical FT programs considering their purposes, target groups, mental health conditions, types of grooming behaviors, and expectations. A school-field link program is also necessary to encourage creating academic, economic, and social values. In conclusion, we propose an integrated model of research protocols of FT research.

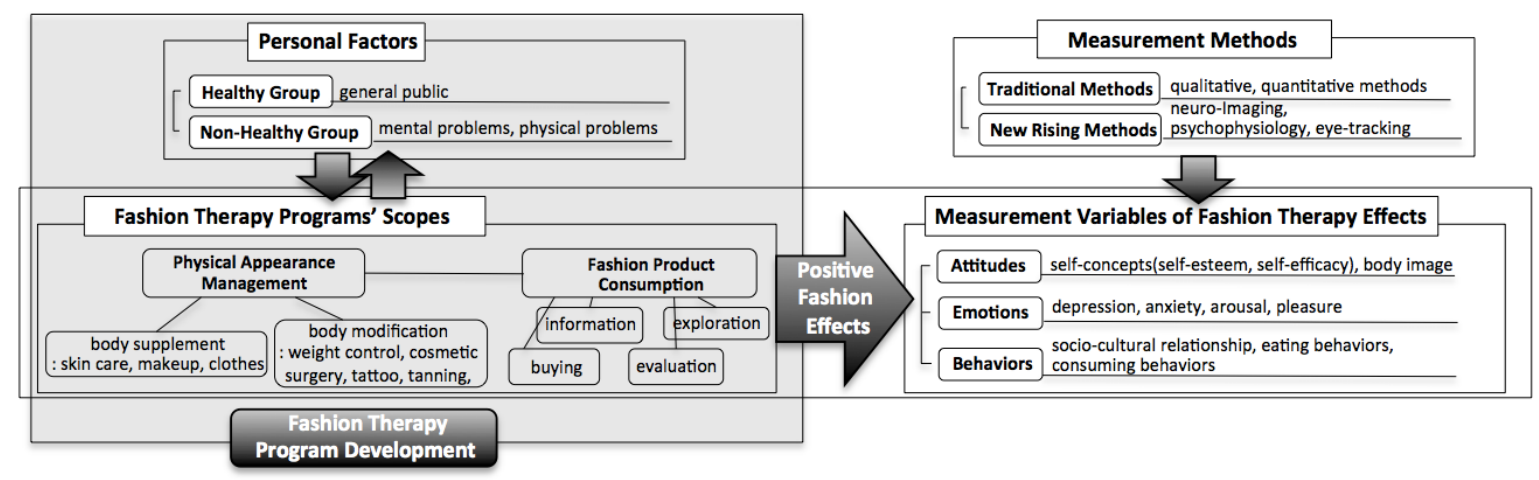

Figure 1. Integrated Model of Research Protocols in Fashion Therapy Research

\section{The value Creations of Fashion Therapy Research}

FT studies could create the academic, economic, and social values. First, in the case of academic values, FT research highlighting positive effects of fashion behaviors could improve scientific values and aid in exploring academic sustainability of CT. Second, FT studies could create economic values to develop practical FT programs which are focused on not only academic purposes but also empirical needs. Particularly, since fashion phenomena are more close to daily lives than art, FT could be applicable to diverse target groups and create more economic values than art therapy. Last, FT research could propose a new era of CT through social value creations. Fashion therapy, including positive fashion effects to improve mental health and enjoyable fashion phenomena in daily lives, would be a trigger of social value creations of CT.

\section{References}

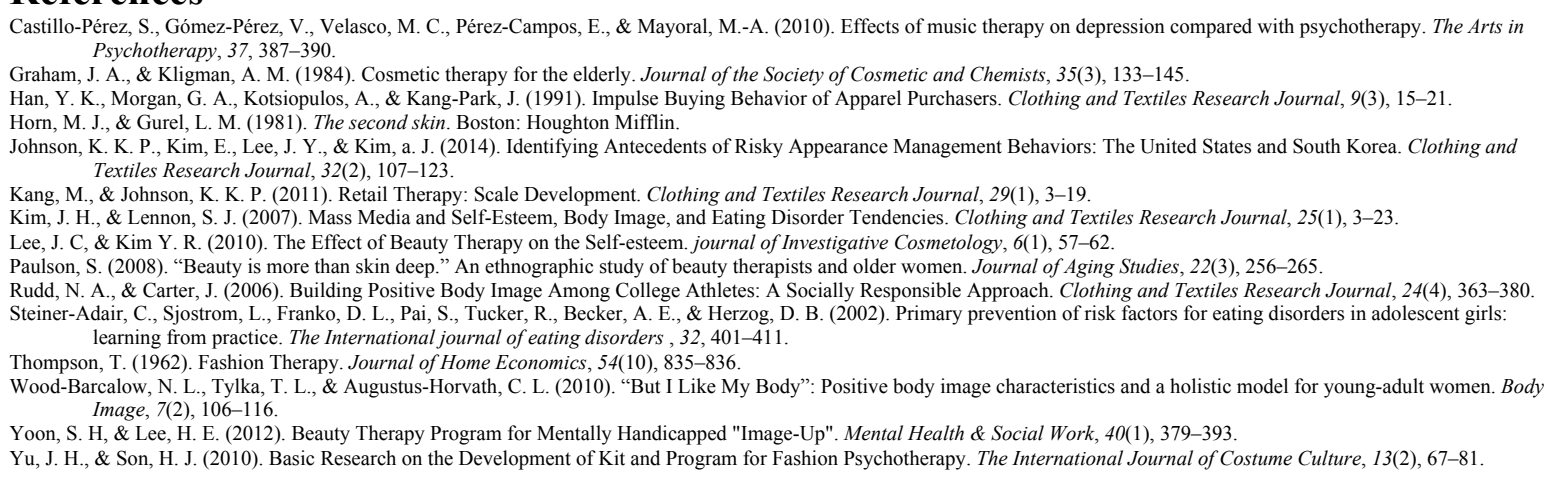

